\title{
Fractional Differentiation-Based Active Contour Model Driven by Local Intensity Fitting Energy
}

\author{
Ming $\mathbf{G u}^{1}$ and Renfang Wang ${ }^{2}$ \\ ${ }^{1}$ Department of Precision Instrument, Tsinghua University, Beijing 100084, China \\ ${ }^{2}$ College of Computer Science and Information Technology, Zhejiang Wanli University, Ningbo 315100, China \\ Correspondence should be addressed to Renfang Wang; renfang_wang@126.com
}

Received 28 October 2015; Revised 28 March 2016; Accepted 19 April 2016

Academic Editor: Weizhong Dai

Copyright (c) 2016 M. Gu and R. Wang. This is an open access article distributed under the Creative Commons Attribution License, which permits unrestricted use, distribution, and reproduction in any medium, provided the original work is properly cited.

\begin{abstract}
A novel active contour model is proposed for segmentation images with inhomogeneity. Firstly, fractional order filter is defined by eight convolution masks corresponding to the image orientation in the eight compass directions. Then, the fractional order differentiation image is obtained and applied to the level set method. Secondly, we defined a new energy functional based on local image information and fractional order differentiation image; the proposed model not only can describe the input image more accurately but also can deal with intensity inhomogeneity. Local fitting term can enhance the ability of the model to deal with intensity inhomogeneity. The defined penalty term is used to reduce the occurrence of false boundaries. Finally, in order to eliminate the time-consuming step of reinitialization and ensure stable evolution of level set function, the Gaussian filtering method is used. Experiments on synthetic and real images show that the proposed model is efficient for images with intensity inhomogeneity and flexible to initial contour.
\end{abstract}

\section{Introduction}

Image segmentation is a fundamental and challenging task in automatic image processing and computer vision. It aims to divide an image into a number of nonoverlapping regions that are more meaningful and are easy to analyze. So, the quality of segmentation directly affects the results of the following high level tasks. Up till now, numerous techniques for image segmentation have been proposed to solve the problem of image segmentation, such as thresholding, edge detection, clustering, region growth, and active contour method. Among those methods, active contour models (ACMs) have been proven to be one of the successful methods for image segmentation. However, images in natural world are often corrupted by intensity inhomogeneity, which makes image difficult to segment accurately.

Since first introduced by Kass et al. [1], ACMs have received more and more attention. The basic idea is that the dynamic curves evolve controlled by an appropriate energy functional and toward the target boundaries. Over the last two decades, many researchers have done great efforts to improve the performance of it and have proposed many different ACMs, which can be categorized into two categories: parametric ACMs and geometric ACMs. However, the parametric ACMs cannot deal with the topological changes like splitting and merging of the evolving curve. In geometric ACMs, this problem can be handled by using the level set method, which is proposed by Osher and Sethian [2]. Differentiating from the pure partial differential equation models whose evolution equation was directly constructed, the variational level set method [3] first defines an energy functional by level set function, and then the evolution equation is obtained via minimizing the energy functional. When the evolution of level set function stops, we can get the final contour represented by the zero level set. One of the most popular variational level set models is the CV model [4], which is built based on the assumption that the intensities in foreground and background keep constant. The CV model has been widely applied to two-phase image segmentation. However, it usually fails to segment images with intensity inhomogeneity. In order to deal with this problem, Li et al. proposed a famous local region-based model named 
LBF $[5,6]$ (local binary fitting). The energy functional is defined by using the local intensity statistical information. Although the LBF model can work well on images with intensity inhomogeneity, it is sensitive to initial contour.

In the image processing, methods based on fractional order differentiation appear to give better performance than traditional ones. In fact, fractional order differentiation, which can date back to three hundred years ago, is a generalization of the ordinary differentiation. Due to more precise derivatives of arbitrary order, fractional order differentiation can provide the best description for many natural phenomena and has been successfully applied to many fields such as signal processing and automatic control $[7,8]$. Though fractional order differentiation has been uses in image denoising [9], image enhancement [10], and image segmentation [11], its application in the area of image processing is just an emerging branch to study.

In the present work, we proposed a novel active contour model based on the fractional order differentiation and level set method to segment images with intensity inhomogeneity. Many natural images are always corrupted by noise and low contrast. The traditional methods based on integer order differentiation improve image visual quality while enhancing noise. However, factional order differentiation can better describe image data than integer order differentiation and get better results. So, we construct filter convolution masks based on the fractional order differentiation. In order to effectively estimate the fractional order differential of digital image and ensure the factional mask windows are invariant to rotation, filter masks are defined in eight directions. And then the filtered image is obtained by the convolution between original image and filter masks. In the level set method, on one hand, local image information plays a vital role in successful segmentation of images with intensity inhomogeneity. So the proposed model defined a new local fitting term to cope with intensity inhomogeneity. On the other hand, false boundaries often appear; therefore, a new penalty term is defined to solve this problem. Finally, the Gaussian filtering method is used to eliminate the time-consuming step of reinitialization and ensure stable evolution of level set function. Experiments on synthetic and real images show that the proposed model is efficient for images with intensity inhomogeneity and flexible to initial contour.

In summary, the main contributions of the paper are as follows. (a) We use the filtered image resulting from fractional order differentiation as a guide image to accurately estimate the local information. The increased quality of this guide image improves the performance of the proposed model. (b) Based on the observation of the Heaviside function, we propose a new penalty function which is used to reduce the occurrence of false boundary. (c) Some numerical experiments are presented to analyze the improvement due to using filter convolution masks based on fractional order.

This paper is organized as follows. In Section 2, we briefly review two classic level set models for image segmentation and indicate their limitations. Section 3 shows a new level set model in detail. Section 4 presents experimental results on real and synthetic images using the proposed model. Finally, conclusions are drawn in Section 5.

\section{Related Work}

2.1. CV Model. Chan and Vese [4] proposed a classic level set model for image segmentation based on the assumption that the input image is piecewise constant. Let $I$ be a given image on the image domain $\Omega$. The $\mathrm{CV}$ model can be expressed as minimization of the following energy functional:

$$
\begin{aligned}
E^{\mathrm{CV}}(\phi)= & \lambda_{1} \int_{\Omega}\left|I(x)-c_{1}\right|^{2} H(\phi(x)) d x \\
& +\lambda_{2} \int_{\Omega}\left|I(x)-c_{2}\right|^{2}(1-H(\phi(x))) d x \\
& +\mu \int_{\Omega}|\nabla H(\phi(x))| d x,
\end{aligned}
$$

where $\mu, \lambda_{1}$, and $\lambda_{2}$ are fixed nonnegative parameters. $\nabla$ is gradient operator. $I(x)$ is the intensity at a point $x$ in $\Omega$. $c_{1}$ and $c_{2}$ are two constants that denote the average intensities in the regions inside and outside the contour $C$, respectively. $\phi$ is the level set function, and $H$ is the Heaviside function.

By minimizing the energy functional (1), we obtain the following formulation:

$$
\begin{aligned}
\frac{\partial \phi}{\partial t}= & \mu \delta(\phi) \operatorname{div}\left(\frac{\nabla \phi}{|\nabla \phi|}\right) \\
& +\delta(\phi)\left[-\lambda_{1}\left(I(x)-c_{1}\right)^{2}+\lambda_{2}\left(I(x)-c_{2}\right)^{2}\right],
\end{aligned}
$$

where div is divergence operator. The two piecewise constants $c_{1}$ and $c_{2}$ are given by

$$
\begin{gathered}
c_{1}=\frac{\int_{\Omega} I(x) H(\phi(x)) d x}{\int_{\Omega} H(\phi(x)) d x}, \\
c_{2}=\frac{\int_{\Omega} I(x)(1-H(\phi(x))) d x}{\int_{\Omega}(1-H(\phi(x))) d x} .
\end{gathered}
$$

In (2), the first term computes the length of contour, which smooths the evolution curves. The second term is the global image fitting force based on the global image information to drive the contour evolution toward the object boundaries. If the intensities inside or outside the contour $C$ are inhomogeneity, $c_{1}$ and $c_{2}$ may be far different from the real image data. So the CV model usually fails to segment images with intensity inhomogeneity.

2.2. LBF Model. To segment images with intensity inhomogeneity, Li et al. proposed the LBF model [5]. The local statistical information is obtained by introducing a kernel function. They defined the energy functional as follows:

$$
\begin{array}{r}
E^{\mathrm{LBF}}=\lambda_{1} \int_{\Omega}\left[\int_{\Omega} K_{\sigma}(x-y)\left|I(y)-f_{1}(x)\right|^{2}\right. \\
\cdot H(\phi(y)) d y] d x+\lambda_{2} \int_{\Omega}\left[\int_{\Omega} K_{\sigma}(x-y)\right.
\end{array}
$$




$$
\begin{aligned}
& \left.\cdot\left|I(y)-f_{2}(x)\right|^{2}(1-H(\phi(y))) d y\right] d x \\
& +\nu \int_{\Omega} \delta(\phi(x))|\nabla \phi(x)| d x+\frac{\mu}{2} \int_{\Omega}(|\nabla \phi(x)| \\
& -1)^{2} d x,
\end{aligned}
$$

where $K_{\sigma}$ is Gaussian kernel with standard deviation $\sigma . \mu \geq$ $0, v \geq 0, \lambda_{1}$, and $\lambda_{2}$ are fixed parameters. $f_{1}$ and $f_{2}$ locally approximate the intensities inside and outside contour in a widow, respectively.

By minimizing the energy functional (4) with regard to level set function $\phi$, we can obtain the following equation:

$$
\begin{aligned}
\frac{\partial \phi}{\partial t}= & \mu\left[\Delta \phi-\operatorname{div}\left(\frac{\nabla \phi}{|\nabla \phi|}\right)\right]+\nu \operatorname{div}\left(\frac{\nabla \phi}{|\nabla \phi|}\right) \\
& -\delta(\phi)\left(\lambda_{1} e_{1}-\lambda_{2} e_{2}\right),
\end{aligned}
$$

where $e_{1}$ and $e_{2}$ are functions:

$$
e_{i}(x)=\int_{\Omega} K_{\sigma}(y-x)\left|I(x)-f_{i}(y)\right|^{2} d y, \quad i=1,2,
$$

with

$$
f_{i}(x)=\frac{\int_{\Omega} K_{\sigma}(x-y) I(y) M_{i}(\phi) d y}{\int_{\Omega} K_{\sigma}(x-y) M_{i}(\phi) d y}, \quad i=1,2,
$$

where $M_{1}(\phi)=H(\phi)$ and $M_{2}=1-H(\phi)$.

In (5), the first term is the penalty term to regularize the level set function, which avoids the initialization step. The second term is the length term and the last term is the local image fitting force that controls the contour evolution. The LBF model can effectively segment inhomogeneity images. However, LBF model is sensitive to initial contour. In other words, it has restrictive requirement for the location of the initial contour.

2.3. Grünwald-Letnikov (GL) Definition. The fractional order differentiation is a generalization of the ordinary differentiation and has started to play a very important role in image processing. The definition of fractional order differentiation is studied by many researchers from different views and more than one exist in literatures. In this paper, we use GL definition, which can be expressed as

$$
t_{0} D_{t}^{v} s(t)=\lim _{h \rightarrow 0} \frac{1}{h^{v}} \sum_{j=0}^{\left[\left(t-t_{0}\right) / h\right]}(-1)^{j}\left(\begin{array}{l}
v \\
j
\end{array}\right) s(t-j h),
$$

where $\left(\begin{array}{c}v \\ j\end{array}\right)=\Gamma(v+1) / \Gamma(j+1) \Gamma(v-j+1), 0<v \leq 1$, and $\Gamma$ is the Gamma function. The explicit numerical approximation can be expressed as

$$
t_{0} D_{t}^{v} s(t) \approx \frac{1}{h^{v}} \sum_{j=0}^{\left[\left(t-t_{0}\right) / h\right]} w_{j}^{(v)} s(t-j h),
$$

where $w_{j}^{(v)}=(-1)^{j}\left(\begin{array}{c}v \\ j\end{array}\right)$, which can be considered as the coefficients of the Taylor series expansions of the corresponding "generating" functions, defined as follows [12]:

$$
\begin{aligned}
W_{1}^{v}(z) & =(1-z)^{v}, \\
W_{2}^{v}(z) & =\left(\frac{3}{2}-2 z+\frac{1}{2} z^{2}\right)^{v}, \\
W_{3}^{v}(z) & =\left(\frac{11}{6}-3 z+\frac{3}{2} z^{2}-\frac{1}{3} z^{3}\right)^{v}, \\
W_{4}^{v}(z) & =\left(\frac{25}{12}-4 z+3 z^{2}-\frac{4}{3} z^{3}+\frac{1}{4} z^{4}\right)^{v} .
\end{aligned}
$$

\section{The Proposed Model}

3.1. Fractional Order Image. In [13], Tian et al. define four directions of fractional order masks: on $x$ - and $y$-axes directions, expressed by $W_{x}^{-}, W_{y}^{-}, W_{x}^{+}$, and $W_{y}^{+}$. It is shown in Figure 1 , where $w_{0}=1, w_{1}=1-v, w_{2}=3 / 2-2 v+(1 / 2) v^{2}$, $w_{3}=11 / 6-3 v+(3 / 2) v^{2}-(1 / 3) v^{3}$, and $w_{4}=25 / 12-4 v+$ $3 v^{2}-(4 / 3) v^{3}+(1 / 4) v^{4}$.

There is no reasonable explanation for defining those coefficients, but we can get inspiration from literature [13]. If the signal duration $\left[t_{0}, t\right]$ is divided equally as $h=1$, then it has $n=\left[\left(t-t_{0}\right) / h\right]^{h=1}=\left[t-t_{0}\right]$. According to (9), the $v$ order fractional differential expression of one-dimensional signal $s(t)$ can be deduced as

$$
\begin{aligned}
\frac{d^{v} s(t)}{d t^{v}} \approx & \frac{1}{h^{v}} \sum_{j=0}^{\left[\left(t-t_{0}\right) / h\right]} w_{j}^{(v)} s(t-j h)=\sum_{j=0}^{n} w_{j}^{(v)} s(t-j) \\
= & s(t)+(-v) s(t-1)+\frac{(-v)(-v+1)}{2} s(t-2) \\
& +\frac{(-v)(-v+1)(-v+2)}{6} s(t-3)+\cdots \\
& +\frac{\Gamma(-v+1)}{(n-1) ! \Gamma(-v+n+1)} s(t-n) .
\end{aligned}
$$

Similarly, for two-dimensional signal $s(x, y)$, the $v$ order fractional partial differential on negative $x$ and $y$ coordinate could be expressed as [14]

$$
\begin{aligned}
\frac{\partial^{v} s(x, y)}{\partial x^{v}} \approx & s(x, y)+(-v) s(x-1, y) \\
& +\frac{(-v)(-v+1)}{2} s(x-2, y) \\
& +\frac{(-v)(-v+1)(-v+2)}{6} s(x-3, y) \\
& +\cdots \\
& +\frac{\Gamma(-v+1)}{(n-1) ! \Gamma(-v+n+1)} s(x-n, y),
\end{aligned}
$$




$$
\begin{aligned}
\frac{\partial^{v} s(x, y)}{\partial y^{v}} \approx & s(x, y)+(-v) s(x, y-1) \\
& +\frac{(-v)(-v+1)}{2} s(x, y-2) \\
& +\frac{(-v)(-v+1)(-v+2)}{6} s(x, y-3) \\
& +\cdots \\
& +\frac{\Gamma(-v+1)}{(n-1) ! \Gamma(-v+n+1)} s(x, y-n) .
\end{aligned}
$$

We can find that the corresponding coefficient of the $v$ order fractional partial differential on negative $x$ and $y$ coordinate is the same. It is also not difficult to find that the bigger the mask size is the higher degree of accuracy that will be obtained, but the computation time will be consuming. Here we select the size of 5 and reset $w_{i}(i=0,1, \ldots, 4)$ defined in $[13]$ as

$$
\begin{aligned}
& w_{0}=1, \\
& w_{1}=-v, \\
& w_{2}=\frac{(-v)(-v+1)}{2}, \\
& w_{3}=\frac{(-v)(-v+1)(-v+2)}{6}, \\
& w_{4}=\frac{(-v)(-v+1)(-v+2)(-v+3)}{24} .
\end{aligned}
$$

The masks shown in Figure 1 can only be used to calculate the fractional order differential in four directions. So it is not effective to estimate the fractional order differential of digital image and less antirotation capability. In order to obtain more accurate estimate and enhance the antirotation capability of the fractional mask windows, in this paper, we use another four direction masks defined in [14]: left downward diagonal, right upward diagonal, left upward diagonal, and right downward diagonal, denoted by $W_{\mathrm{LDD}}$, $W_{\mathrm{RUD}}, W_{\mathrm{LUD}}$, and $W_{\mathrm{RDD}}$. This is illustrated in Figure 2.

By multiplying the coefficient values on eight directions of the mask and the corresponding input $I$, we can obtain eight images, denoted by $I_{x}^{-}, I_{y}^{-}, I_{x}^{+}, I_{y}^{+}, I_{\mathrm{LDD}}, I_{\mathrm{RUD}}, I_{\mathrm{LUD}}$, and $I_{\mathrm{RDD}}$. According to (12), it easily can be seen that, among these nonzero coefficients, only the coefficient of the first term is the constant 1; the others are nonzero and functions with respect to fractional order $v$. The sum of those masks is not equal to 1 . This may change the distribution of pixel value and lead to side effects. Here, normalization method is used to solve this problem. the fractional order image $D^{v} I$ can be calculated according to the following formula:

$$
\begin{aligned}
& D^{v} I \\
& =\frac{\left(I_{x}^{-}+I_{y}^{-}+I_{x}^{+}+I_{y}^{+}+I_{\mathrm{LDD}}+I_{\mathrm{RUD}}+I_{\mathrm{LUD}}+I_{\mathrm{RDD}}\right)}{\operatorname{sum}},
\end{aligned}
$$

where sum $=8 *\left(\sum_{i=0}^{4} w_{i}\right)$.
3.2. Level Set Formulation. In the practice, the level set function is usually defined as follows:

$$
\phi(x)= \begin{cases}-c_{0}, & \text { if } x \text { is inside } C \\ 0, & \text { if } x \in C \\ c_{0}, & \text { if } x \text { is outside } C\end{cases}
$$

where $c_{0}$ is a positive constant. In a numerical implementation, the Heaviside function $H(\phi)$ and the corresponding Dirac function $\delta(\phi)$ are replaced with a smooth function expressed as

$$
\begin{aligned}
& H_{\varepsilon}(\phi)=\frac{1}{2}\left(1+\frac{2}{\pi} \arctan \left(\frac{\phi}{\varepsilon}\right)\right), \\
& \delta_{\varepsilon}(\phi)=\frac{d}{d \phi} H_{\varepsilon}(\phi)=\frac{1}{\pi}\left(\frac{\varepsilon}{\varepsilon^{2}+\phi^{2}}\right) .
\end{aligned}
$$

From (16), we can find the following: (1) if $\phi=0$, then $H_{\varepsilon}(\phi)=0.5$; (2) if $\phi>0$, then $0.5<H_{\varepsilon}(\phi)<1$; (3) if $\phi<0$, then $0<H_{\varepsilon}(\phi)<0.5$. Now, we define a penalty term:

$$
E^{P}(\phi)=\int_{\Omega} H_{\varepsilon}(\phi)\left(1-H_{\varepsilon}(\phi)\right) d x
$$

when minimizing $E^{P}, H_{\varepsilon}(\phi) \rightarrow 0$ or 1 , so $\phi$ is forced to not be equal to 0 . In other words, fewer points are marked as boundary points. So the defined penalty term can reduce the occurrence of false boundary. As discussed above, local image information plays a vital role in successful segmentation of images with intensity inhomogeneity. The local image information is used to define the fractional order based fitting term, defined as follows:

$$
\begin{aligned}
E^{F}= & \lambda_{1} \int_{\Omega}\left(D^{v} I(x)-m_{1}\right)^{2} H_{\varepsilon}(\phi(x)) d x \\
& +\lambda_{2} \int_{\Omega}\left(D^{v} I(x)-m_{2}\right)^{2}\left(1-H_{\varepsilon}(\phi(x))\right) d x,
\end{aligned}
$$

where $m_{1}$ and $m_{2}$ are defined as follows:

$$
\begin{aligned}
& m_{1}=\operatorname{mean}\left(D^{v} I \in\left(\{x \in \Omega \mid \phi(x)<0\} \cap W_{k}(x)\right)\right), \\
& m_{2}=\operatorname{mean}\left(D^{v} I \in\left(\{x \in \Omega \mid \phi(x)>0\} \cap W_{k}(x)\right)\right),
\end{aligned}
$$

where $W_{k}$ is a rectangular window function such as constant window or a truncated Gaussian window. In this paper, a truncated Gaussian window $K_{\sigma}(x)$ with the deviation $\sigma$ is selected. $m_{1}$ and $m_{2}$ are local averages of the input image intensities inside and outside the contour $C$. In this paper, the 


\begin{tabular}{|c|c|c|c|c|}
\hline 0 & 0 & $w_{4}$ & 0 & 0 \\
\hline 0 & 0 & $w_{3}$ & 0 & 0 \\
\hline 0 & 0 & $w_{2}$ & 0 & 0 \\
\hline 0 & 0 & $w_{1}$ & 0 & 0 \\
\hline 0 & 0 & $w_{0}$ & 0 & 0 \\
\hline
\end{tabular}

(a)

\begin{tabular}{|c|c|c|c|c|}
\hline 0 & 0 & $w_{0}$ & 0 & 0 \\
\hline 0 & 0 & $w_{1}$ & 0 & 0 \\
\hline 0 & 0 & $w_{2}$ & 0 & 0 \\
\hline 0 & 0 & $w_{3}$ & 0 & 0 \\
\hline 0 & 0 & $w_{4}$ & 0 & 0 \\
\hline
\end{tabular}

(c)

\begin{tabular}{|c|c|c|c|c|}
\hline 0 & 0 & 0 & 0 & 0 \\
\hline 0 & 0 & 0 & 0 & 0 \\
\hline$w_{4}$ & $w_{3}$ & $w_{2}$ & $w_{1}$ & $w_{0}$ \\
\hline 0 & 0 & 0 & 0 & 0 \\
\hline 0 & 0 & 0 & 0 & 0 \\
\hline
\end{tabular}

(b)

\begin{tabular}{|c|c|c|c|c|}
\hline 0 & 0 & 0 & 0 & 0 \\
\hline 0 & 0 & 0 & 0 & 0 \\
\hline$w_{0}$ & $w_{1}$ & $w_{2}$ & $w_{3}$ & $w_{4}$ \\
\hline 0 & 0 & 0 & 0 & 0 \\
\hline 0 & 0 & 0 & 0 & 0 \\
\hline
\end{tabular}

(d)

FIGURE 1: Masks of four directions: (a) $W_{x}^{-}$, (b) $W_{y}^{-}$, (c) $W_{x}^{+}$, and (d) $W_{y}^{+}$.

regularization term $E^{R}=\int_{\Omega}\left|\nabla H_{\varepsilon}(\phi(x))\right| d x$ is also used. The energy functional of the proposed model is defined as follows:

$$
\begin{aligned}
E= & E^{F}+\nu E^{P}+\mu E^{R} \\
= & \lambda_{1} \int_{\Omega}\left(D^{v} I(x)-m_{1}\right)^{2} H_{\varepsilon}(\phi(x)) d x \\
& +\lambda_{2} \int_{\Omega}\left(D^{v} I(x)-m_{2}\right)^{2}\left(1-H_{\varepsilon}(\phi(x))\right) d x \\
& +\nu \int_{\Omega} H_{\varepsilon}(\phi(x))\left(1-H_{\varepsilon}(\phi(x))\right) d x \\
& +\mu \int_{\Omega}\left|\nabla H_{\varepsilon}(\phi(x))\right| d x .
\end{aligned}
$$

Minimizing the energy functional (21) by the steepest descent method, we obtain the following formulation:

$$
\begin{aligned}
\frac{\partial \phi}{\partial t} & =\delta_{\varepsilon}(\phi) \\
\cdot & {\left[-\lambda_{1}\left(D^{v} I(x)-m_{1}\right)^{2}+\lambda_{2}\left(D^{v} I(x)-m_{2}\right)^{2}\right] } \\
& +\nu \delta_{\varepsilon}(\phi)\left(2 H_{\varepsilon}(\phi)-1\right)+\mu \delta_{\varepsilon}(\phi) \operatorname{div}\left(\frac{\nabla \phi}{|\nabla \phi|}\right),
\end{aligned}
$$

where $H_{\varepsilon}(\phi)$ and $\delta_{\varepsilon}(\phi)$ are defined in (16) and (17), respectively.

3.3. Implementation. The reinitialization is necessary to prevent the level set function from becoming too flat or steep in the traditional level set methods [15]. However, implementation of this method is quite complex and time-consuming. To overcome this problem, many methods [16-18] are proposed. Based on the scale-space theory [19], the Gaussian filtering method is proposed in [16]. In this paper, the Gaussian filtering method is used to regularize the level set function to eliminate the time-consuming step of reinitialization. The main steps of the proposed model are summarized as follows:

(1) Initialize the level set function according to (15).

(2) Compute the fractional order differentiation image according to (14).

(3) Compute $m_{1}$ and $m_{2}$ using (20).

(4) Update the level set function $\phi$ according to (22).

(5) Regularize the level set function $\phi=G_{\xi} * \phi$ using Gaussian filtering method, where $\xi$ is the standard deviation.

(6) Extract the zero level set from the level set function $\phi_{t}(x)$, if the level set evolution terminates at time $t$. 


\begin{tabular}{|c|c|c|c|c|}
\hline 0 & 0 & 0 & 0 & $w_{0}$ \\
\hline 0 & 0 & 0 & $w_{1}$ & 0 \\
\hline 0 & 0 & $w_{2}$ & 0 & 0 \\
\hline 0 & $w_{3}$ & 0 & 0 & 0 \\
\hline$w_{4}$ & 0 & 0 & 0 & 0 \\
\hline
\end{tabular}

(a)

\begin{tabular}{|c|c|c|c|c|}
\hline$w_{4}$ & 0 & 0 & 0 & 0 \\
\hline 0 & $w_{3}$ & 0 & 0 & 0 \\
\hline 0 & 0 & $w_{2}$ & 0 & 0 \\
\hline 0 & 0 & 0 & $w_{1}$ & 0 \\
\hline 0 & 0 & 0 & 0 & $w_{0}$ \\
\hline
\end{tabular}

(c)

FIGURE 2: Masks of four diagonal directions: (a) $W_{\mathrm{LDD}}$

$W_{\mathrm{LDD}}$, (b) $W_{\mathrm{RUD}}$,

(d)

\begin{tabular}{|c|c|c|c|c|}
\hline$w_{0}$ & 0 & 0 & 0 & 0 \\
\hline 0 & $w_{2}$ & 0 & 0 & 0 \\
\hline 0 & 0 & $w_{2}$ & 0 & 0 \\
\hline 0 & 0 & 0 & $w_{3}$ & 0 \\
\hline 0 & 0 & 0 & 0 & $w_{4}$ \\
\hline
\end{tabular}

\begin{tabular}{|c|c|c|c|c|}
\hline 0 & 0 & 0 & 0 & $w_{4}$ \\
\hline 0 & 0 & 0 & $w_{3}$ & 0 \\
\hline 0 & 0 & $w_{2}$ & 0 & 0 \\
\hline 0 & $w_{1}$ & 0 & 0 & 0 \\
\hline$w_{0}$ & 0 & 0 & 0 & 0 \\
\hline
\end{tabular}

(b)

\section{Experimental Result}

This section shows the experimental results of the proposed model. All the experiments are carried out by Matlab (R2010b) in the PC with Dual $2.83 \mathrm{GHz}$ processor. The parameters are set as $v=0.2, v=200, \lambda_{1}=\lambda_{2}=1, \varepsilon=1.0$, $\sigma=5, \Delta t=0.1, \xi=0.5, c_{0}=1$, and $\mu=0.01 \times 255 \times 255$.

4.1. Application on Synthetic and Real Images. Figure 3 shows the results for synthetic images. The first row shows the original images with initial contour and the second row shows the segmentation results. These images are corrupted by intensity inhomogeneity. Figure 4 shows the segmentation result for four real images: plane, red-crowned crane, X-ray, and MRI. It can be seen that the proposed model obtain satisfactory segmentation results owing to considering the local fractional order image information, which ensures the proposed model effectively extracts the object boundaries.

4.2. Comparisons with the CV Model and the LBF Model. The parameters of the CV model are set as: $\lambda_{1}=\lambda_{2}=1$, $\mu=0.001 \times 255 \times 255$. The parameters of the LBF model are set as: $\lambda_{1}=\lambda_{2}=1, \nu=0.001 \times 255 \times 255, \sigma=3, \mu=1$. Figure 5 shows the segmentation results of the CV model and the proposed model. The two segmented images contain several objects with different intensities and the intensity of some objects is similar to the background intensity. The segmentation results are illustrated in the last column, we can find the proposed model can successfully extract the boundaries of all objects. In contrast, the CV model fails to detect all objects. Figure 6 shows the segmentation of four images with intensity inhomogeneity. We employ the same initial contour in order to compare the proposed model with LBF model. From the results, we can see the LBF model failed to segment all images with this initialization. However, the proposed model has desirable performance on these images.

4.3. Robustness to Initial Contours. To further evaluate the proposed model, we apply it and the LBF model to segment two vessel images with different initial contours. Figure 7 shows the segmentation results of the LBF model and the proposed model with the different initial contours. The first row shows the five different initializations. The second row is the results of the LBF model. The third row is the results of the proposed model. From the segmentation results, we can see that the LBF model is sensitive to initial contour, but the proposed model is not.

4.4. About the Parameter v. Figure 8 shows the obtained images with different parameter $v$ and the corresponding segmentation results. The first row from left to right shows original image: $v=0.2, v=0.4$, and $v=0.6$, respectively. 

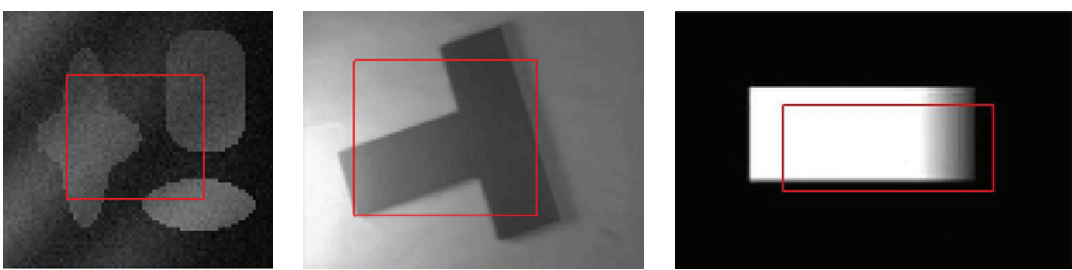

(a)
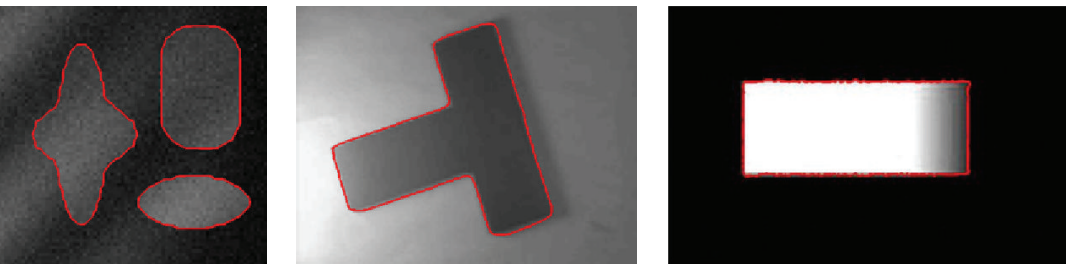

(b)
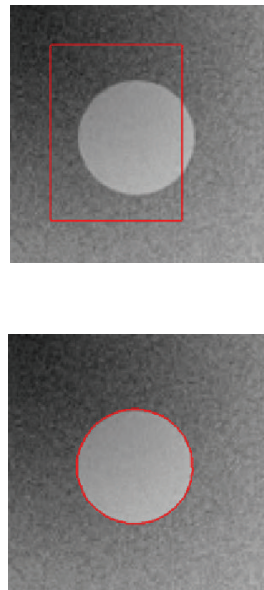

FIGURE 3: Results of our proposed method for synthetic images: (a) initial contours; (b) final contours.
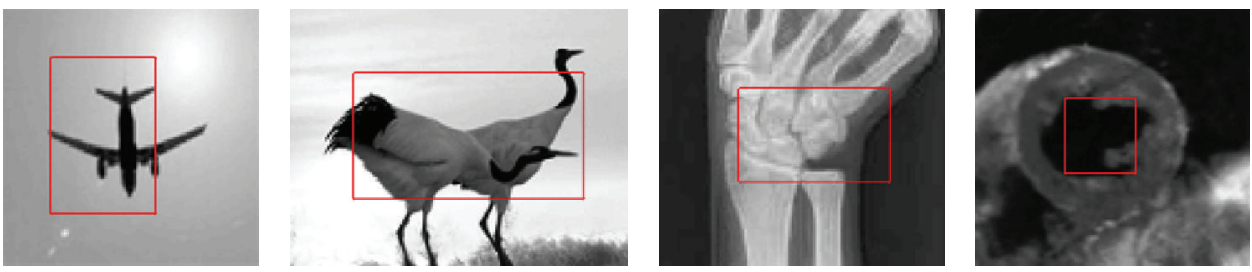

(a)
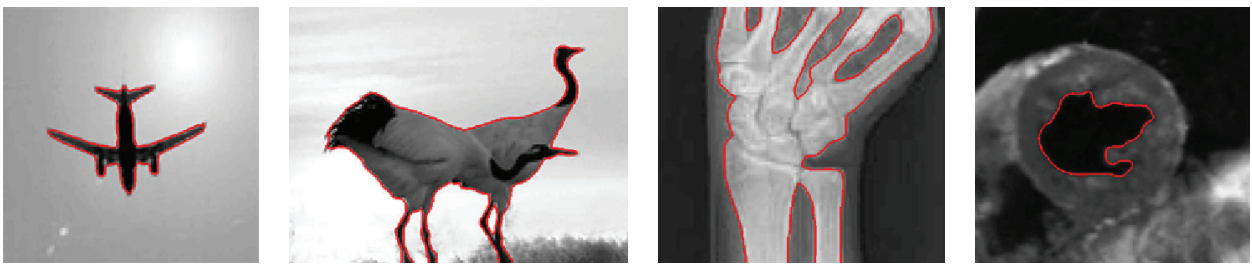

(b)

FIGURE 4: Results of our proposed method for real images: (a) initial contours; (b) final contours.
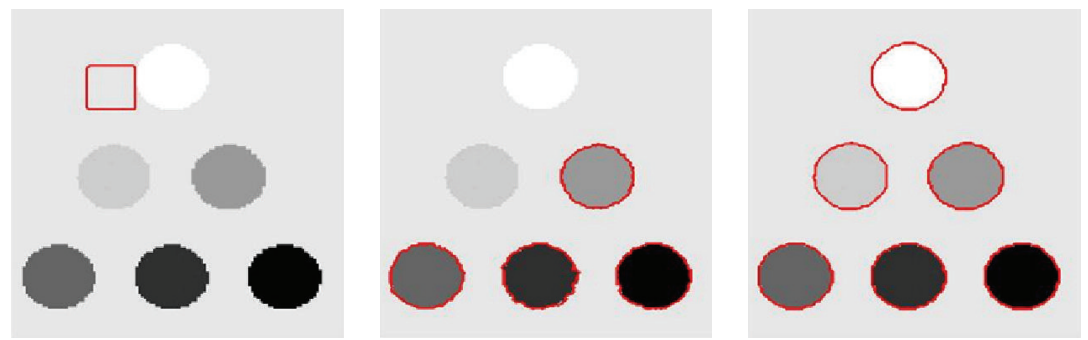

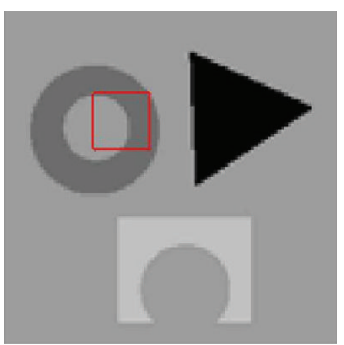

(a)

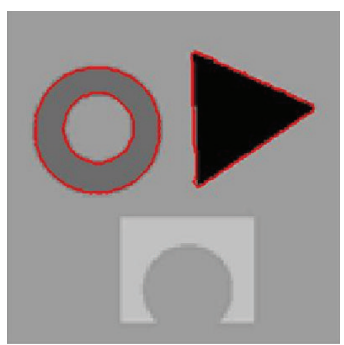

(b)

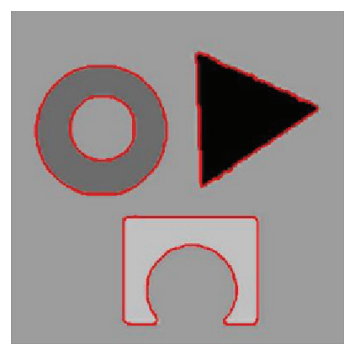

(c)

FIgURE 5: Comparison of the proposed model with the CV model: (a) initial contour; (b, c) results of CV model and the proposed model. 

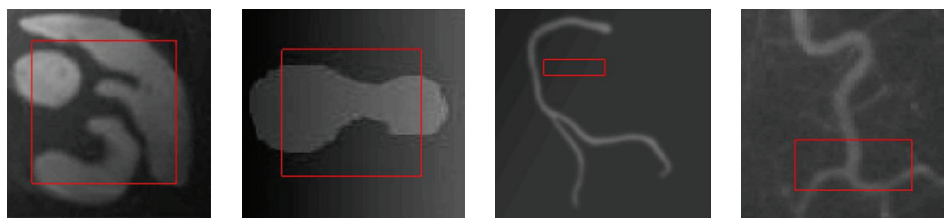

(a)
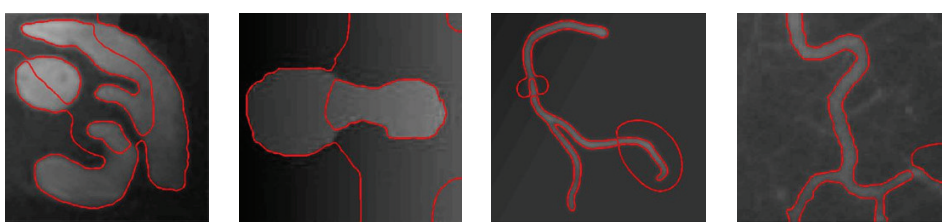

(b)
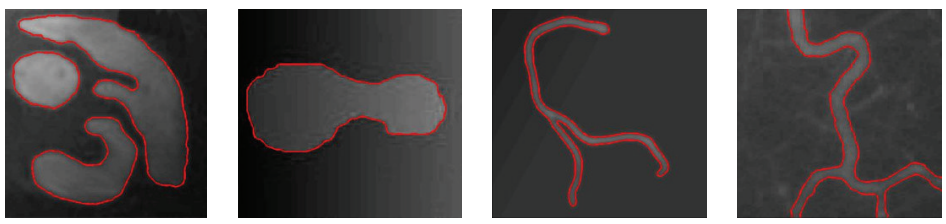

(c)

FIGURE 6: Segmentation results of LBF and the proposed model: (a) initial contour; (b) the results of LBF; (c) the proposed model.
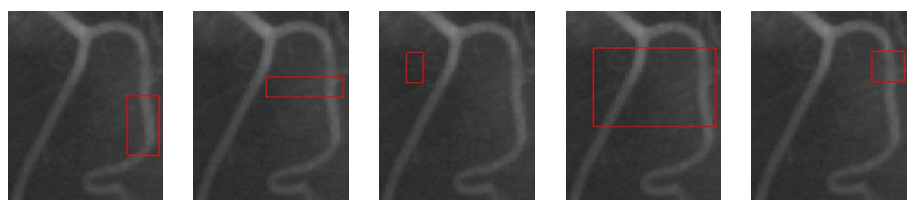

(a)
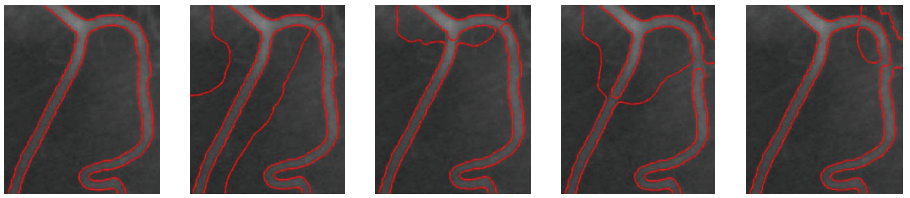

(b)
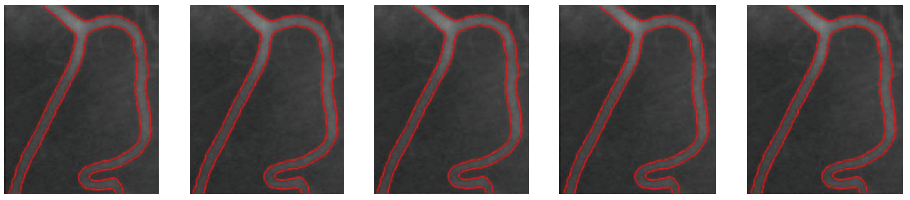

(c)

Figure 7: Segmentation results of LBF and the proposed model with different contours: (a) initial contours; (b) the results of LBF model; (c) the results of the proposed method.

We can find that contrast is enhanced gradually, However, noise is also enhanced. For the original image, we can see the level set curve disappeard. This is mainly due to low contrast. When $v=0.4,0.8$, the method also fails to get correct segmentation results because of the influence of noise. However, the proposed model gets desirable result by setting $v=0.2$. In fact, the parameter $v$ should be set according to image. Fortunately, $v=0.2$ works well on all images in this paper.

\section{Conclusion}

This paper proposed a novel active contour model based on the local image information and fractional order differentiation image. The local information based fitting term helps the proposed model to deal with images with intensity inhomogeneity. The new penalty term can reduce the occurrence of the false boundaries and makes the model more robust. Specially, the filter masks can effectively improve the quality 

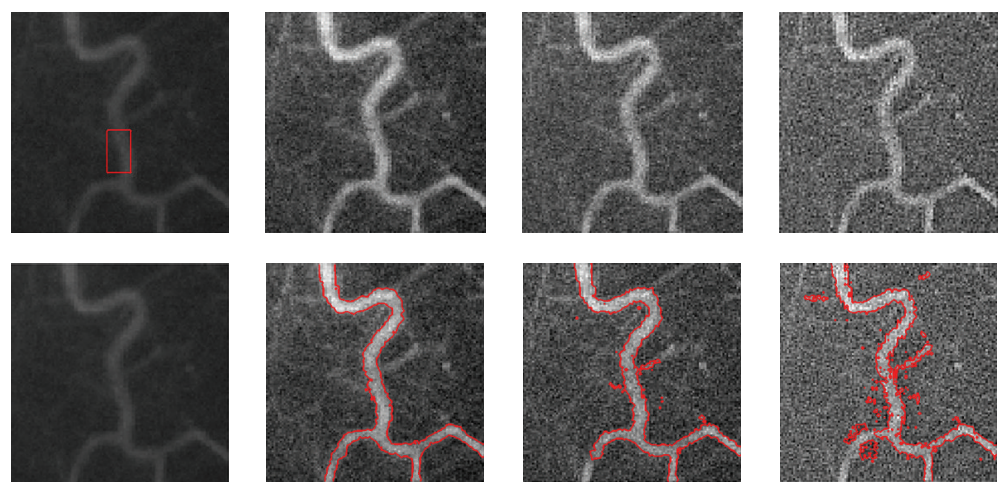

(a)

(b)

(c)

(d)

Figure 8: Segmentation results based on different $v$.

of the low-contrast images. The proposed model can tackle intensity inhomogeneity and is less sensitive to the initial contour. Extensive experiments demonstrated the advantages of the proposed model over the CV model and the LBF model.

\section{Competing Interests}

The authors declare that they have no competing interests.

\section{Acknowledgments}

This work is supported in part by the Project of the Science and Technology Plan for Zhejiang Province (Grant no. 2016C31084) and the Ningbo Natural Science Foundation (Grant no. 2016A610228).

\section{References}

[1] M. Kass, A. Witkin, and D. Terzopoulos, "Snakes: active contour models," International Journal of Computer Vision, vol. 1, no. 4, pp. 321-331, 1988.

[2] S. Osher and J. A. Sethian, "Fronts propagating with curvaturedependent speed: algorithms based on Hamilton-Jacobi formulations," Journal of Computational Physics, vol. 79, no. 1, pp. 1249, 1988.

[3] D. Mumford and J. Shah, "Optimal approximations by piecewise smooth functions and associated variational problems," Communications on Pure and Applied Mathematics, vol. 42, no. 5, pp. 577-685, 1989.

[4] T. F. Chan and L. A. Vese, "Active contours without edges," IEEE Transactions on Image Processing, vol. 10, no. 2, pp. 266-277, 2001.

[5] C. Li, C.-Y. Kao, J. C. Gore, and Z. Ding, "Implicit active contours driven by local binary fitting energy," in Proceedings of the IEEE Computer Society Conference on Computer Vision and Pattern Recognition (CVPR '07), pp. 1-7, Minneapolis, Minn, USA, June 2007.

[6] C. Li, C.-Y. Kao, J. C. Gore, and Z. Ding, "Minimization of region-scalable fitting energy for image segmentation," IEEE Transactions on Image Processing, vol. 17, no. 10, pp. 1940-1949, 2008.
[7] A. Carpinteri, B. Chiaia, and P. Cornetti, "Static-kinematic duality and the principle of virtual work in the mechanics of fractal media," Computer Methods in Applied Mechanics and Engineering, vol. 191, no. 1-2, pp. 3-19, 2001.

[8] P. Zavada, "Relativistic wave equations with fractional derivatives and pseudodifferential operators," Journal of Applied Mathematics, vol. 2, no. 4, pp. 163-197, 2002.

[9] J. Bai and X.-C. Feng, "Fractional-order anisotropic diffusion for image denoising," IEEE Transactions on Image Processing, vol. 16, no. 10, pp. 2492-2502, 2007.

[10] H. A. Jalab and R. W. Ibrahim, "Texture enhancement based on the Savitzky-Golay fractional differential operator," Mathematical Problems in Engineering, vol. 2013, Article ID 149289, 8 pages, 2013.

[11] Y.-F. Pu and J.-L. Zhou, "A novel approach for multi-scale texture segmentation based on fractional differential," International Journal of Computer Mathematics, vol. 88, no. 1, pp. 58-78, 2011.

[12] K. B. Oldham and J. Spanier, The Fractional Calculus, Academic Press, New York, NY, USA, 1974.

[13] D. Tian, D. Xue, D. Cheri, and S. Sun, "A fractional-order regulatory $\mathrm{CV}$ model for brain $\mathrm{MR}$ image segmentation," in Proceedings of the 25th Chinese Control and Decision Conference (CCDC '13), pp. 37-40, IEEE, May 2013.

[14] Y. Pu, W. Wang, J. Zhou, Y. Wang, and H. Jia, "Fractional differential approach to detecting textural features of digital image and its fractional differential filter implementation," Science in China Series F: Information Sciences, vol. 51, no. 9, pp. 1319-1339, 2008.

[15] R. Malladi, J. A. Sethian, and B. C. Vemuri, "Shape modeling with front propagation: a level set approach," IEEE Transactions on Pattern Analysis and Machine Intelligence, vol. 17, no. 2, pp. 158-175, 1995.

[16] K. Zhang, L. Zhang, H. Song, and W. Zhou, "Active contours with selective local or global segmentation: a new formulation and level set method," Image and Vision Computing, vol. 28, no. 4, pp. 668-676, 2010.

[17] K. Zhang, L. Zhang, H. Song, and D. Zhang, "Reinitializationfree level set evolution via reaction diffusion," IEEE Transactions on Image Processing, vol. 22, no. 1, pp. 258-271, 2013. 
[18] C. Li, C. Xu, C. Gui, and M. D. Fox, "Distance regularized level set evolution and its application to image segmentation," IEEE Transactions on Image Processing, vol. 19, no. 12, pp. 3243-3254, 2010.

[19] P. Perona and J. Malik, "Scale-space and edge detection using anisotropic diffusion," IEEE Transactions on Pattern Analysis and Machine Intelligence, vol. 12, no. 7, pp. 629-639, 1990. 


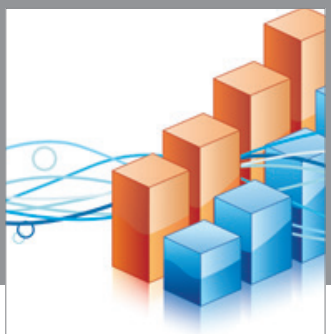

Advances in

Operations Research

vatem alat4

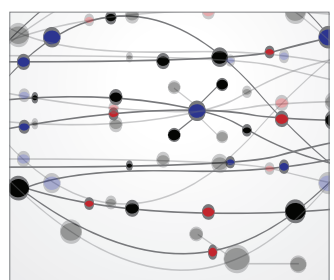

\section{The Scientific} World Journal
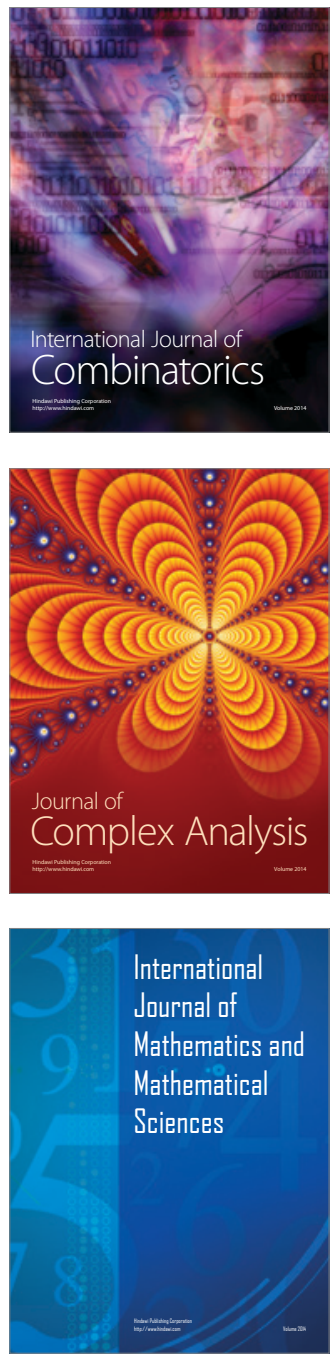
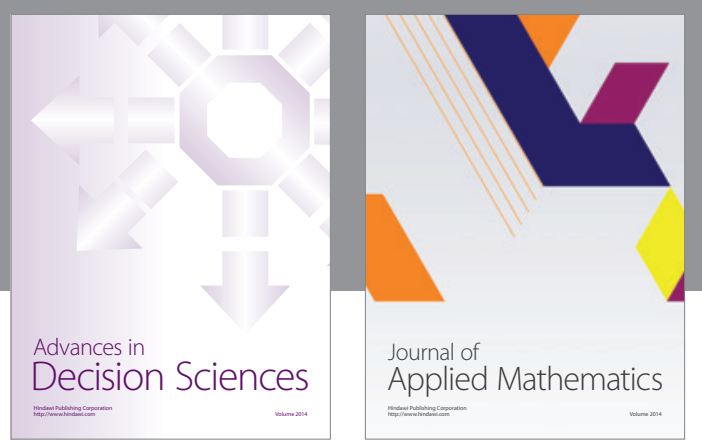

Algebra

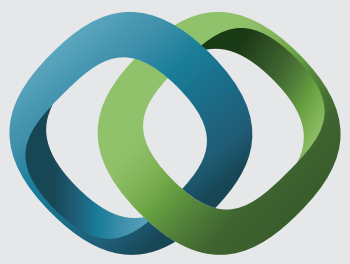

\section{Hindawi}

Submit your manuscripts at

http://www.hindawi.com
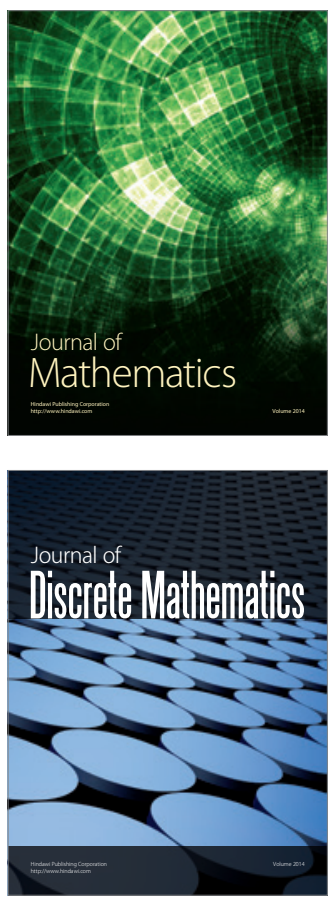

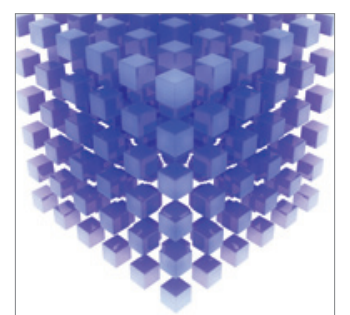

Mathematical Problems in Engineering
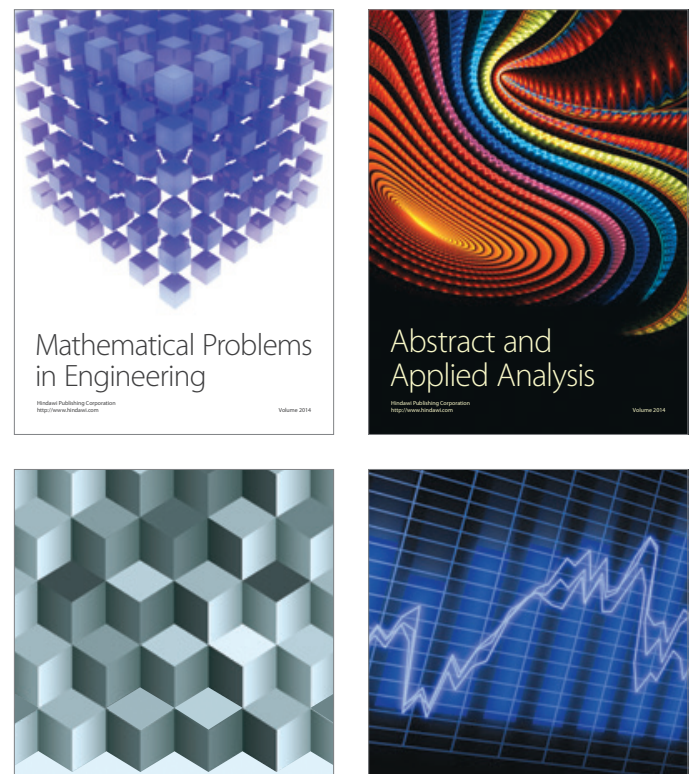

Journal of

Function Spaces

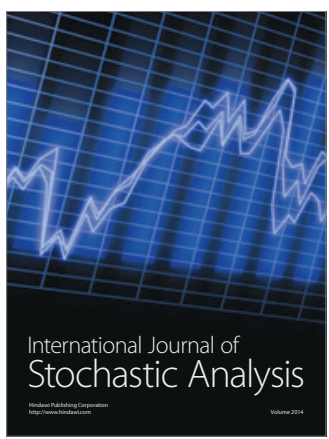

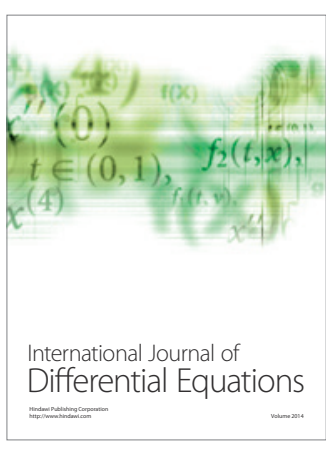
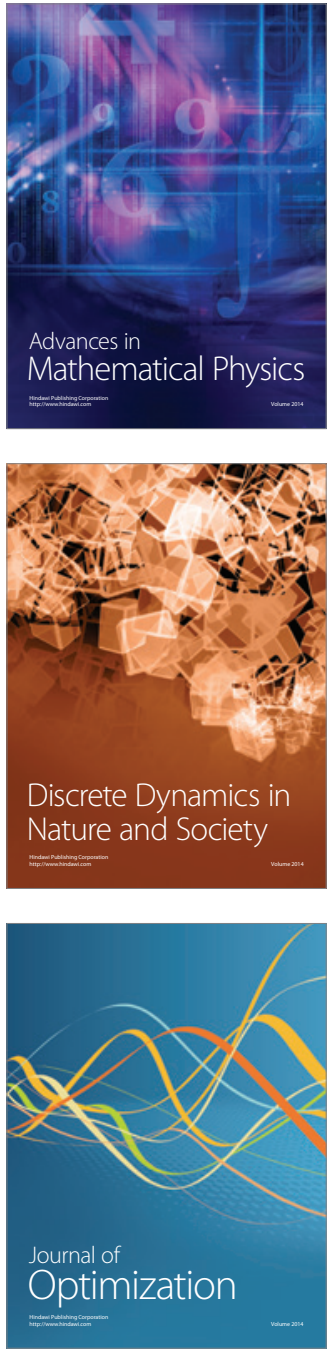